\title{
Dynamic Reliable Location Aware Routing Protocols for Mobile Wireless Sensor Network
}

\author{
Simerjeet Sharma \\ M.Tech Student \\ Department of Computer Science and \\ Engineering SGGSW University, \\ Fatehgarh Shaib
}

\author{
Navpreet Kaur \\ Assistant Prof. \\ Department of Computer Science and \\ Engineering SGGSW University, \\ Fatehgarh Shaib
}

\begin{abstract}
Energy efficiency, network lifetime, data transmission, endto-end delay, reliable routing protocols for mobility centric and mobility of Cluster Head are the major parameters used for wireless sensor network (WSN), and are maintained by various of protocols. Existing cluster-based mobile routing protocols such as LEACH, LEACH-Mobile, LEACH-Mobile Enhanced, CBR-Mobile, LFCP-MWSN consider energy efficiency of sensor node. These protocols allocate the extra timeslots using time division multiple access (TDMA) scheme to accommodate nodes that enter a cluster because of mobility and thus increase the end-to-end delay. In this study a new ILFCP-MWSN protocol is proposed based on mobility of the cluster head. The results revealed that ILFCP-MWSN protocol is more efficient in terms of Energy Consumptions, Remaining Energy and Approximate Number of Data Transmissions than those of the existing LEACH-M, LEACHME, LFCP-MWSN protocols.
\end{abstract}

\section{Keywords}

LEACH; Clustering Methods; Wireless Sensor Network (WSN).

\section{INTRODUCTION}

The Smart Dust Project at University of California, Berkeley $[14,15,16]$ and WINS Project at UCLA [1, 17], are two of the research projects attempting to build such low-cost and extremely small (approximately 1 cubic millimeter) sensors. An ad-hoc wireless network is much inexpensive but less reliable and accurate sensors can be used in a wide variety of commercial and military applications.

Wireless sensor networks (WSNs) consist of hundreds and thousands of unattended, resource-constraint and low-energy sensor nodes designing energy efficient routing protocols is significantly important. Clustering-based routing protocols are more useful in the context of energy efficiency where several sensor nodes in the communication range of one another form a cluster $[1,2]$. Most clustering protocols of WSN in the literature are designed for static sensor nodes. Thus, these protocols do not work for WSN applications that require mobile sensor nodes, such as habitat monitoring, wild life monitoring, target tracking and battlefield surveillance [5].

Wireless sensor network (WSN) is widely considered as one of the most important technologies for the twenty-first century [21]. In the past decades, it has received tremendous attention from both academia and industry all over the world. A WSN typically consists of a large number of low-cost, low-power, and multifunctional wireless sensor nodes, with sensing, wireless communications and computation capabilities [20, 23]. These sensor nodes communicate over short distance via a wireless medium and collaborate to accomplish a common task, for example, environment monitoring, military surveillance, and industrial process control [24]. The basic philosophy behind WSNs is while the capability of each individual sensor node is limited, the aggregate power of the entire network is sufficient for the required mission. In many WSN applications, the deployment of sensor nodes is performed in an ad hoc fashion without careful planning and engineering. Once deployed, the sensor nodes must be able to autonomously organize themselves into a wireless communication network. Sensor nodes are battery-powered and are expected to operate without attendance for a relatively long period of time. In most cases, it is very difficult and even impossible to change or recharge batteries for the sensor nodes. WSNs are characterized with denser levels of sensor node deployment, higher unreliability of sensor nodes, and sever power, computation, and memory constraints. Thus, the unique characteristics and constraints present many new challenges for the development and application of WSNs. Wireless sensor networks (WSNs) consist of hundreds and thousands of unattended, resource-constraint and low-energy sensor nodes designing energy efficient routing protocols is significantly important. Clustering-based routing protocols are more useful in the context of energy efficiency where several sensor nodes in the communication range of one another forms a cluster. Each cluster has a cluster head $(\mathrm{CH})$, which coordinates all the nodes of a cluster. There may be a number of base stations (BS) also known as sink in a WSN that communicate with other networks $[1,2]$.

Moreover, these protocols do not support localization of sensor nodes but only assume that each node know their location, which make these protocols inefficient. For instance, low energy adaptive clustering hierarchy (LEACH) Protocol is a standard static clustering protocol of WSN [3]. LEACH is enhanced as LEACH-Mobile [4], LEACH-MobileEnhancement [5], cluster based routing protocol for mobile nodes in wireless sensor network (CBR Mobile-WSN) [1] and location aware fault tolerant clustering protocol for mobile WSN (LFCP-MWSN) to support mobility of sensor nodes. This is also not considered energy efficient since a large number of messages are transmitted to form a cluster. To alleviate this problem we proposed a method in which cluster head $(\mathrm{CH})$ is mobile. To overcome the limitation of existed protocols such as Energy Consumption, Approximate Number of Transmission and cluster head movement, a new protocol is used. Improved Location aware Fault Tolerant Clustering Protocol for Mobile WSN (ILFCP-MWSN) proposed for overcome the limitation and comparison is also done in this how it give better result as compared to other protocols.

\section{RELATED WORKS}

Low energy adaptive clustering Protocol (LEACH) Durresia, A., Paruchuri, V., Barolli, L. [2] conveyed that various clustering techniques were used by different protocols and applications to increase scalability and reduce delays in sensor networks. CP was a lightweight protocol that did not require any neighborhood information and imposed lesser 
communication overhead. Clustering Protocol (CP), a novel protocol for clustering a sensor network was an extension of the Covered Problem encountered in geometry. Theprotocol was performed in an asynchronous anddistributed manner by each node in the network. CP imposed lesser communication overhead and the efficiency of CP remained very high even in large networks.

Li Qing, Qingxin Zhu, Mingwen Wang [11] conveyed that the clustering Algorithm was a kind of key technique used to reduce energy consumption. It had increased the scalability and lifetime of the network. Energy-efficient clustering protocols should be designed for the characteristic of heterogeneous wireless sensor networks. A new distributed energy-efficient clustering scheme for heterogeneous wireless sensor networks, which was called DEEC. In DEEC, every sensor node independently elected itself as a cluster-head based on its initialized energy and residual energy. To control the energy expenditure of nodes by mean of adaptive approach, DEEC had used the average energy of the network as the reference energy. DEEC did not require any global knowledge of energy at every election round. DEEC performed well in multi-level heterogeneous wireless sensor networks.

Wendi B. Heinzelman, Anantha P. Chandrakasan, and HariBalakrishnan [12]conveyed that the designing protocol architectures for wireless micro sensor networks was important to consider the function of the application, the need for eased of deployment, and the severed energy constraints of the nodes. Low-energy adaptive clustering hierarchy (LEACH), protocol architecture for micro sensor networks that combined the ideas of energy-efficient cluster-based routing and media accessed together with application-specific data aggregate into achieved good performance in terms of system lifetime, latency, and application-perceived quality. LEACH included a new, distributed cluster formation technique that enabled self-organizationof large numbers of nodes, algorithms for adapted clustersand rotated cluster head positions to evenly distribute the energyload among all the nodes, and techniques to enabled distributedsignal processing to save communication resources. Results of experiment showed that LEACH provides the high performance needed under the tight constraints of the wireless channel. LEACHMobile (LEACH-M) routing protocol where cluster formation and $\mathrm{CH}$ selection mechanism was same as LEACH. LEACH$\mathrm{M}$ ensured the communication of a node with a $\mathrm{CH}$ even if node was in motion by transmitting data request packet from $\mathrm{CH}$ to the sensor node in its allocated timeslot using TDMA scheme. LEACH-M handled node mobility by assuming that the $\mathrm{CHs}$ were stationary.

LEACH-Mobile-Enhanced (LEACH-ME) where a node with the minimal mobility factor was selected as a $\mathrm{CH}$, if the residual energy of the node was not below a threshold value. They calculated mobility factor based on the number of times a node moves from a cluster to another cluster. Since mobility factor was a function of distance among nodes it was calculated by multiplying node's velocity with the time required to move a node from a position to another. For example, node $\mathrm{i}$ can made use of IDs of all other nodes it heard and calculate $\operatorname{dij}(t) \operatorname{asdij}(t)=$ RadioVelocity*|t2 - t1|, where at time $\mathrm{t} 1$ node $\mathrm{i}$ broadcast its ID and at time $\mathrm{t} 2 \mathrm{it}$ received the ID of node j.Kumar, G.S., Vinu , M.V., Athithan, P.G., Jacob, K.P. [3]conveyed that the routing algorithms currently available were assumed that the sensor nodes are stationary. The LEACH protocol could beenhanced to handle mobility modulation. The LEACH-M protocol where nodes isolated due to mobility from the cluster were reconnected to a newcluster through appropriate mechanism. The LEACH-ME protocol followed the same reconnection mechanism for the isolated node. It used the concept ofremoteness for electing the cluster head. It was cleared that to achieve the level of extra performance, energy dissipation needs to be sacrificed at a tolerable level.

CBR-M was an adaptive protocol that avoids wastage of timeslots and hence, ensures efficient bandwidth utilization. Each $\mathrm{CH}$ kept some free timeslots to enable other incoming mobile nodes from other clusters to join its cluster. A CH sent data request message to the non- $\mathrm{CH}$ nodes and if the $\mathrm{CH}$ did not receive data from a member, the packet was considered to be lost and the $\mathrm{CH}$ discards the nodes membership, at the end of the frame. Awwad, S.A.B., Ng, C.K., Noordin, N.K., Rasid, M.F.A [1] conveyed that CBR Mobile-WSN change TDMA scheduling adaptively according to traffic and mobility characteristics. The protocol sent data to cluster heads in an efficient manner based on received signal strength. The performance of CBR Mobile-WSN protocol was evaluated using MATLAB and it had been observed that the CBR Mobile-WSN protocol reduces the packet loss by $25 \%$ compared to LEACH Mobile protocol. Adaptive scheduling with round free cluster head protocol called CBR MobileWSN. This protocol reduced the effect of mobility by decreasing packet loss by changing the TDMA scheduling adaptively and using round free cluster head. This protocol was also energy aware, as it reduced the energy consumption by transmitting with low transmission with minimal amount of energy power based on the received signal strength of data request message.

All sensors were mobile. Once a node was selected as a $\mathrm{CH}$, it remains in the same cluster. Initially, all sensors have the same energy. A node in each cluster was equipped with GPS and work only for localization. This node was known as anchors node. Sensors were heterogeneous in terms of their roles since they worked as anchor nodes, cluster heads, and cluster members. LFCP-MWSN Protocol used C programming language.

L.Karim, N.Nasser [8] conveyed cluster-based mobile routing protocols such as LEACH-Mobile, LEACH-Mobile-Enhanced and CBR-Mobile consider only the energy efficiency of the sensor nodes. Reliability of routing protocols by incorporated fault tolerance scheme was significantly important to identify the failure of data link and sensor nodes and recovered the transmission path. That LFCP-MWSN protocol was more efficient in terms of energy consumptions, network lifetime and data transmissions than LEACH-M and LEACH-ME protocols. Moreover, LFCP-MWSN detected the failure of sensor nodes. In LFCP-MWSN protocol a node with the least mobility factor was selected as a $\mathrm{CH}$, and then the $\mathrm{CH}$ did not move out of the cluster in the current round.

\section{PROPOSED MOBILE ROUTING PROTOCOL}

We proposed an IFCP-MWSN protocol that supports mobility of sensor nodes, sensor localization and mobility of cluster head. Cluster mobility is considered one of the most important aspect in WSN applications and unique in this proposed protocols of WSN. In this section, we presented the working principle of our proposed ILFCP-MWSN in several phases. The proposed algorithm works with the following assumptions. 
- All sensors are mobile.

- $\quad$ Once a node is selected as a $\mathrm{CH}$, Cluster Head $(\mathrm{CH})$ is mobile.

- Initially, all sensors have the same energy.

- A node in each cluster is equipped with GPS and work only for localisation. This node is known as an anchors node.

- Sensors are heterogeneous in terms of their roles since they work as anchor nodes, cluster heads, and cluster members.

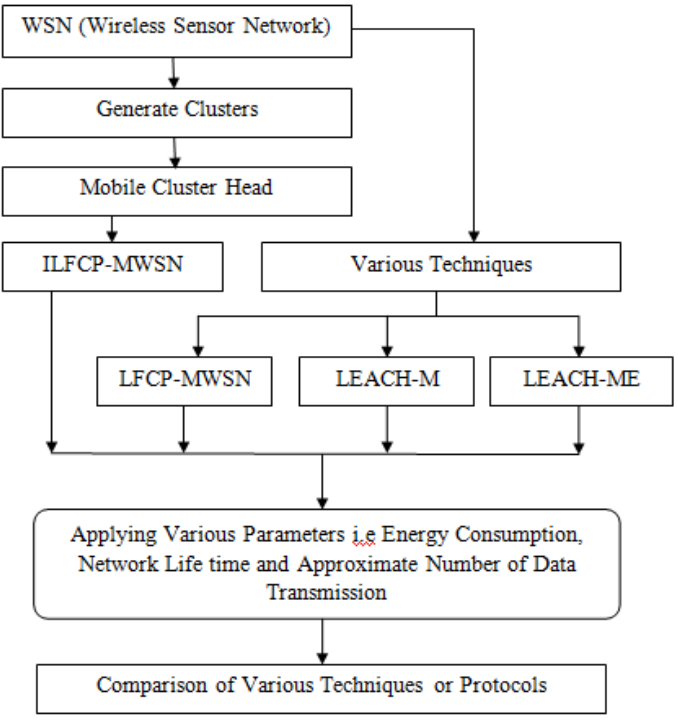

Flow Chart of Proposed Work

\section{EXPERIMENTAL DESIGN}

The following subsections present the simulation setup andresults and performance evaluation of the existing andproposed ILFCP-MWSN protocols.

\section{Simulation Setup}

We simulate the proposed ILFCP-MWSN protocol using Cprogramming language. A simulation model is implementedwith a network of size $100 \times 100 \mathrm{~m}$. BS is place at the coordinate $(50,50)$. Table 2 shows the network parametersand their respective values that are used in the simulation.Users are allowed to input the number of nodes (maximum 100), clusters (maximum 10), rounds, and cluster formation phases in our simulator. For networks with a fixed numberof clusters and nodes, we change the number of rounds at different runs of the simulation.

\subsection{Simulation Results}

We measure the performance of the ILFCP-MWSN protocoland compare it with the existing ILFCP-MWSN, LEACH-M and LEACH-MEmobile clustering protocols in terms of network energyconsumptions, lifetime (remaining network energy), and approximate number of communications. The performance of our proposed protocol is also compared with the existing static clustering protocols, LEACH and DSC, in terms of network energy consumptions and lifetime. Network energy consumption is defined as the total energyconsumed by all the sensors nodes for routing data over acertain period of time. Network energy consumptions also reflect the lifetime of the network, that is, the remaining network energy since network energy consumptions is inversely proportional to the network lifetime. Another important parameter to measure the performance of a mobile routing protocol is the packet delivery ratio which is defined as the total number of packets received at the BS to the total number of packets transmitted by the senders. However, we plan to measure the packet delivery ratio and end-to end delay of ILFCP-MWSN as a part of the future works. We run the simulation over a number of rounds, where around consists of a number of frames. Each non $\mathrm{CH}$ node has a timeslot allocated to it in a frame. The number of frames in a frame is dynamically adjusted based on theresidual energy of networks at the end of each round.

Table 2: Simulation Parameters and their Values

\begin{tabular}{ll}
\hline Parameter & Value \\
\hline Network Size & $100 * 100 \mathrm{~m}$ \\
Number of Nodes & maximum 100 \\
Number of Clusters & maximum 10 \\
Base Station Position & {$[50,50]$} \\
Data Packet size & $256 \mathrm{bits}$ \\
Transmitting Energy Consumption & $50 \mathrm{~nJ} / \mathrm{bit}$ \\
Energy Consumption in Free Space & $0.01 \mathrm{~nJ} / \mathrm{bit} / \mathrm{m}^{2}$ \\
Initial Node Energy & $2 \mathrm{~J}$ \\
Cluster Head Probability & 0.01 \\
Sensor Nodes Velocity & $0.01 \mathrm{~m} / \mathrm{s}$
\end{tabular}

\subsection{Comparison with Existing Mobile Routing Protocols}

Figures 1-5 illustrate the performance of ILFCPMWSN protocol in terms of network energy consumptions, network lifetime and number of communications over a number of rounds. Figure 1 shows that how we generate cluster it shows the cluster on which position it generate. Figure 2 shows the generation of cluster head it tell us that how cluster head it generate on different number of rounds, cluster head is mobile so it also show the mobility of the cluster head. Figure 3shows that the network energy consumptions for ILFCP-MWSN protocolare much less than that of LFCP-MWSN, LEACH-M and LEACH-ME protocols. Hence, the network lifetime of ILFCP-MWSN is more than that of the LFCP-MWSN, LEACH-M and LEACH-ME protocols (Figure 4). This is because in ILFCP-MWSN protocol, each member node of a cluster sends either asmall sized special packet that consumes much less energy or the subscribed data event at its allocated timeslot usingTDMA scheme. It is more likely that the events of interest do not occur frequently (or at every timeslot). Number of communications that take place in ILFCP-MWSN protocol is also lower than that of the other three protocols as shown in Figure 5.One of the possible explanations is that although we are expecting that mobility of each node and mobility of cluster head are equally likely, in realitythis is not the case. 


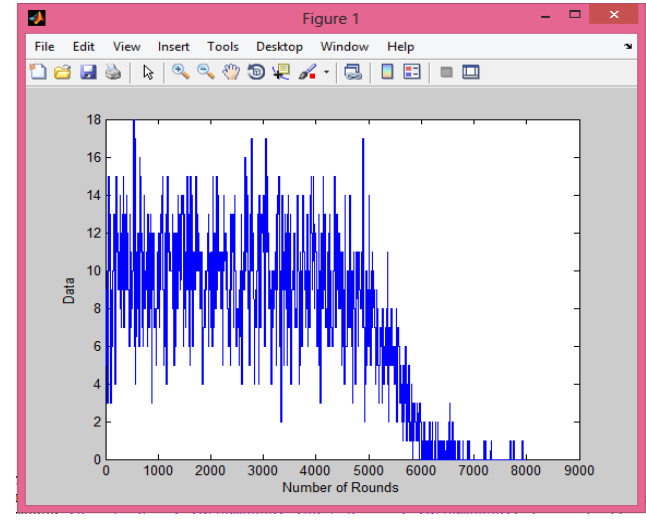

Figure 1: Generating the clusters

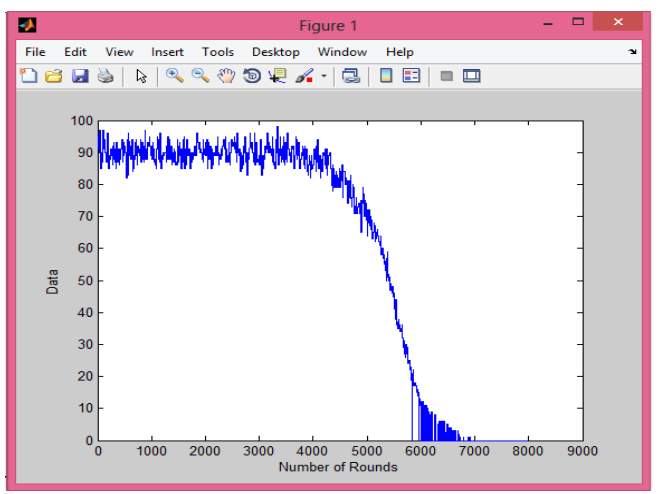

Figure 2: Generating Cluster Head

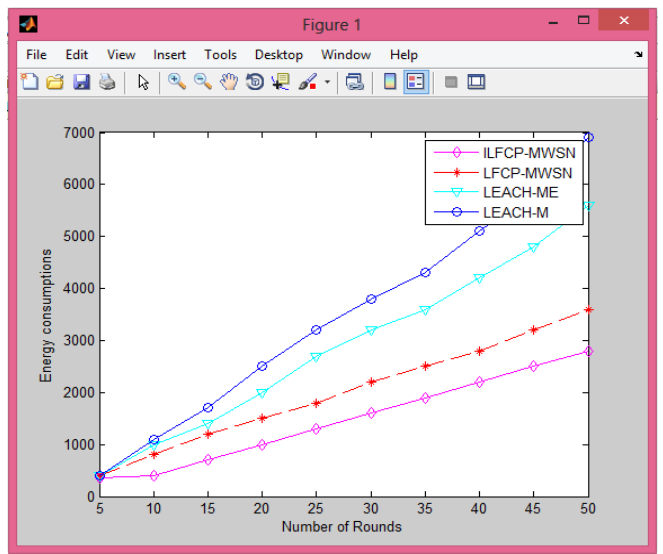

Figure 3: Comparison of Network Energy Consumptions with mobile routing protocols

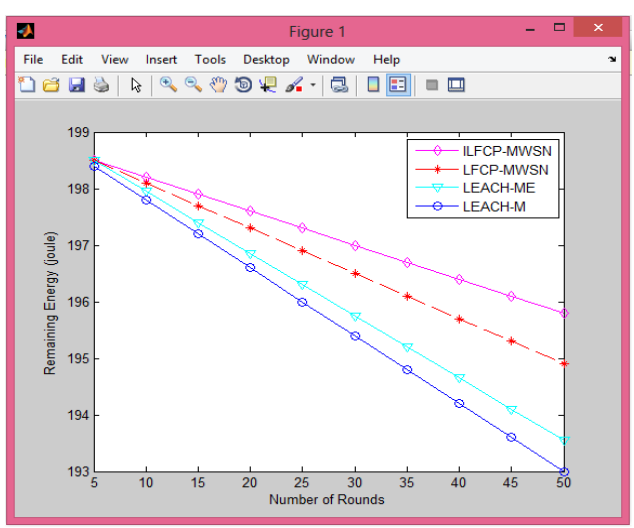

Figure 4: Comparison of Network Lifetime with mobile routing protocols

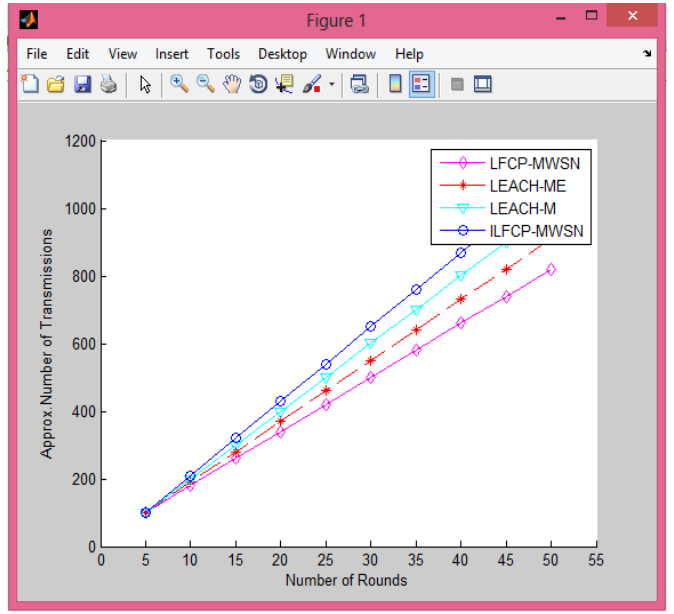

Figure 5: Comparison of Number of Communication with mobile routing protocols

\section{CONCLUSION}

ILFCP-MWSN that supports mobility of sensor nodes, sensors localization and mobility of cluster head $(\mathrm{CH})$. Sensors localization and Cluster Head mobility are considered the most important features for WSN applications and unique in this proposed protocol as compared to most existing mobile routing protocols of WSN. ILFCP-MWSN uses special packets, which are sent by member nodes of a cluster to $\mathrm{CH}$ when member nodes have no sensed event to send to $\mathrm{CH}$ but these special packets allow the ILFCP-MWSN protocol to detect the mobility and failure of member nodes of a cluster. Simulation results showed that ILFCP-MWSN protocol is more efficient in terms of energy consumptions, network lifetime and data transmissions than those of the existing LEACH-M, LEACH-ME protocols and LFCP-MWSN. ILFCP-MWSN detects the failure of sensor nodes. In ILFCPMWSN protocol, we had considered that once a node with the least mobility factor is selected as a $\mathrm{CH}$, then the $\mathrm{CH}$ will move out of the cluster in the current round. The future work suggests a protocol that the aim to consider other factors such as variable timeslots duration for nodes at different clusters to reduce the end-to-end delay of ILFCP-MWSN protocol and enhance ILFCP-MWSN protocol for future.

\section{REFERENCES}

[1] Awwad, S.A.B., Ng, C.K., Noordin, N.K., Rasid, M.F.A., "Cluster based routing protocol for mobile nodes in wireless sensor network", International Conference on Collaborative Technologies and Systems (CTS), pp. 233-241, 2009.

[2] Durresia, A., Paruchuri, V., Barolli, L., "Clustering protocol for sensor networks",20th International Conference on Advanced Information Networking and Applications (AINA), Vol. 2, 2006.

[3] Kumar, G.S., Vinu , M.V., Athithan, P.G., Jacob, K.P., "Routing protocol enhancement for handling node mobility in wireless sensor networks", IEEE Region Conference, 2009.

[4] Zheng, G.-p., Zhou, Y., "An energy-aware cluster protocol for wireless sensor networks", Second International Conference on Innovative Computing, Information and Control (ICICIC), Vol. 7, NO. 10, pp. 473-473, 2012. 
[5] Huang, B., Hao, F., Zhu, H., Tanabe, Y., Baba, T., "Lowenergy static clustering scheme for wireless sensor network", International Conference on Wireless Communications, Networking and Mobile Computing (WiCOM), 2006

[6] Bajaber, F., Awan, I., "Dynamic/static clustering protocol for wireless sensor network", Second UKSIM European Symp. on Computer Modeling and Simulation IEEE, Vol. 8, pp. 524-529, 2008.

[7] Kim, D.-S., Chung, Y.-J., "Self-organization routing protocol supporting mobile nodes for wireless sensor network", First International Multi-Symp. On Computer and Computational Sciences, pp. 221-234, 2006.

[8] L.Karim, N.Nasser, "Reliable location-aware routing protocol for mobile wireless sensor network", International Conference on the Institution of Engineering and Technology, Vol. 6, No. 14, pp. 21492150,2012

[9] SeemaBandyopadhyay and Edward J. Coyle, "An Energy Efficient Hierarchical Clustering Algorithm for Wireless Sensor Networks", IEEE Region Conference, pp. 17131723,2003

[10] I.F. Akyildiz, W. Su, Y. Sankarasubramaniam, E. Cayirci ,"Wireless sensor networks: a survey", Elsevier Science B.V., Vol. 38, No. 4, pp. 393-422, 2002.

[11] Li Qing, Qingxin Zhu, Mingwen Wang, "Design of a distributed energy-efficient clustering algorithm for heterogeneous wireless sensor networks", Elsevier B.V. All rights reserved, pp. 2230-2237, 2006.

[12] Wendi B. Heinzelman, Anantha P. Chandrakasan, and HariBalakrishnan, "An Application-Specific Protocol Architecture for Wireless Microsensor Networks", IEEE transaction on wireless communications, Vol. 1, NO. 4, pp. 660-670, 2002

[13] Shashidhar Rao Gandham, MilindDawande, Ravi Prakashand S. Venkatesan, "Energy Efficient Schemes for Wireless Sensor Networks with Multiple Mobile Base Stations", IEEE Conference, pp. 377-381, 2003.

[14] KisukKweon, HojinGhim, Jaeyoung Hong and Hyunsoo Yoon, "Grid-Based Energy-Efficient Routing from Multiple Sources to Multiple Mobile Sinks in Wireless Sensor Networks", IEEE Conference, pp. 1-5, 2009.

[15] G. J. Pottie and W. J. Kaiser, "Wireless Integrated Network Sensors", Communications of the ACM, Vol. 43, No. 5, pp 51-58, 2000

[16] B. Warneke, M. Last, B. Liebowitz, Kristofer and S. J. Pister, "Smart Dust: Communicating with a CubicMillimeter Computer", Computer Magazine, Vol. 34, No. 1, pp 44-51, 2001.

[17] J. M. Kahn, R. H. Katz and K. S. J. Pister, "Next Century Challenges: Mobile Networking for Smart Dust", In the 5th Annual ACM/IEEE International Conference on Mobile Computing and Networking (MobiCom 99), pp. 271-278, 1999.

[18] V. Hsu, J. M. Kahn, and K. S. J. Pister, "Wireless Communications for Smart Dust", Electronics Research Laboratory Technical Memorandum M98/2 Conference, 1998.
[19] S.K. Singh, M.P. Singh, and D.K. Singh, "A survey of Energy-Efficient Hierarchical Cluster-based Routing in Wireless Sensor Networks", International Journal of Advanced Networking and Application (IJANA), Vol. 02, No. 02, pp.570-580, 2010.

[20] S.K. Singh, M.P. Singh, and D.K. Singh, "Energyefficient Homogeneous Clustering Algorithm for Wireless Sensor Network", International Journal of Wireless \& Mobile Networks (IJWMN), Vol. 2, No. 3, pp. 49-61, 2010.

[21] Jamal Al-Karaki, and Ahmed E. Kamal, "Routing Techniques in Wireless Sensor Networks: A Survey", IEEE Communications Magazine, Vol 11, No. 6, pp. 628, 2004.

[22] Kemal Akkaya and Mohamed Younis, "A Survey on Routing Protocols for Wireless Sensor Networks", Ad hoc Networks, Vol. 3, No. 3, pp. 325-349, 2005

[23] N. Bulusu, J. Heidemann, and D. Estrin, "GPS-less Low Cost Outdoor Localization for Very Small Devices", IEEE Personal Communication Magazine, Vol. 7, No. 5, pp. 28-34, 2000.

[24] B. Nath and D. Niculescu, "Routing on a curve", ACM SIGCOMM Computer Communication Review, Vol. 33, No.1, pp. 155-160, 2003.

[25] V. Rodoplu and T. H. Meng, "Minimum energy mobile wireless networks", IEEE Journal on Selected Areas in Communications, Vol. 17, No. 8, pp. 1333-1344,1999.

[26] J. Kulik, W. Heinzelman, and H. Balakrishnan, "Negotiation-based protocols for disseminating information in wireless sensor networks", Wireless Networks, Vol. 8, No. 2/3, pp. 169-185, 2002.

[27] C. Intanagonwiwat, R. Govindan, D. Estrin, J. Heidemann, and F. Silva, "Directed diffusion for wireless sensor networking", IEEE/ACM Transactions on Networking, Vol. 11., No. 1, pp. 2-16, 2003.

[28] A. Savvides, C-C Han, aind M. Srivastava, Dynamic - ne-grained localization in Ad-Hoc networks of sensors," Proceedings of the Seventh ACM Annual International Conference on Mobile Computing and Networking (MobiCom) Conference, pp.166-179,2001.

[29] S. Capkun, M. Hamdi, J. Hubaux,"GPS-free positioning in mobile ad-hoc networks", Proceedings of the 34th Annual Hawaii International Conference on System Sciences, pp. 3481-3490, 2001.

[30] Y. Xu, J. Heidemann, D. Estrin, IGeography-informed Energy Conservation for Ad-hoc Routing," In Proceedings of the Seventh Annual ACM/IEEE International Conference on Mobile Computing and Networking, pp. 70-84, 2001.

[31] S. Lindsey, C. S. Raghavendra, and K. M. Sivalingam, "Data gathering algorithms in sensor networks using energy metrics", IEEE Transactions on Parallel and Distributed Systems, Vol. 13, No. 9, pp. 924-935, 2002.

[32] M. Chu, H. Haussecker, and F. Zhao, "Scalable information-driven sensor querying and routing for ad hoc heterogeneous sensor networks", International Journal of High Performance ComputingApplications, Vol. 16, No. 3, pp. 293-313,2002. 
[33] AnkitaJoshi , Lakshmi Priya.M, "A Survey of Hierarchical Routing Protocols in Wireless Sensor Network", MES Journal of Technology and Management Conference, pp. 67-71, 2004.

[34] Shio Kumar Singh, M P Singh, and D K Singh, "Routing Protocols in Wireless Sensor Networks -A Survey", International Journal of Computer Science \& Engineering Survey (IJCSES) Vol.1, No.2, 2010.
[35] Shital Y. Agrawal, Prof. C. M. Raut, “A Survey on Location Based Routing Protocols for Wireless Sensor Network", International Journal of Emerging Technology and Advanced Engineering, Volume 3, No. 9, 2013.

[36] Ravinder Kaur, Dr.Trilok Chand Aseri, "Routing Protocols for Mobile Wireless Sensor Networks",International Journal of Software and Web Sciences (IJSWS) in Conference, pp. 31-35, 2013. 